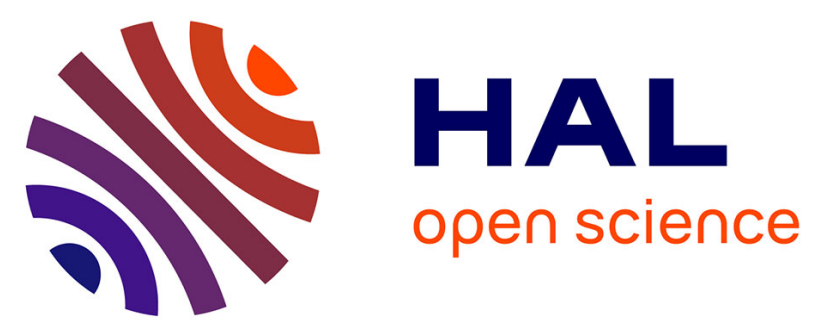

\title{
A High Voltage High Frequency Resonant Inverter for Supplying DBD Devices with Short Discharge Current Pulses
}

\author{
Xavier Bonnin, Julio Brandelero, Nicolas Videau, Hubert Piquet, Thierry \\ Meynard
}

\section{To cite this version:}

Xavier Bonnin, Julio Brandelero, Nicolas Videau, Hubert Piquet, Thierry Meynard. A High Voltage High Frequency Resonant Inverter for Supplying DBD Devices with Short Discharge Current Pulses. IEEE Transactions on Power Electronics, 2014, vol. 29 ( $\mathrm{n}^{\circ}$ 8), pp. 4261-4269. 10.1109/TPEL.2013.2295525 . hal-01407703

\section{HAL Id: hal-01407703 https://hal.science/hal-01407703}

Submitted on 2 Dec 2016

HAL is a multi-disciplinary open access archive for the deposit and dissemination of scientific research documents, whether they are published or not. The documents may come from teaching and research institutions in France or abroad, or from public or private research centers.
L'archive ouverte pluridisciplinaire HAL, est destinée au dépôt et à la diffusion de documents scientifiques de niveau recherche, publiés ou non, émanant des établissements d'enseignement et de recherche français ou étrangers, des laboratoires publics ou privés. 


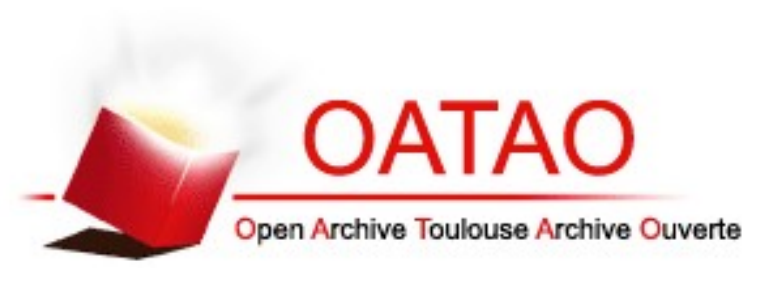

\section{Open Archive Toulouse Archive Ouverte (OATAO)}

OATAO is an open access repository that collects the work of Toulouse researchers and makes it freely available over the web where possible.

This is an author-deposited version published in: http://oatao.univ-toulouse.fr/ Eprints ID: 16523

To link to this article : DOI: 10.1109/TPEL.2013.2295525

URL : http://dx.doi.org/10.1109/TPEL.2013.2295525

To cite this version: Bonnin, Xavier and Brandelero, Julio and Videau, Nicolas and Piquet, Hubert and Meynard, Thierry A High Voltage High Frequency Resonant Inverter for Supplying DBD Devices with Short Discharge Current Pulses. (2014) IEEE Transactions on Power Electronics, vol. 29 ( $\mathrm{n}^{\circ} 8$ ). pp. 4261-4269. ISSN 0885-8993

Any correspondence concerning this service should be sent to the repository administrator: staff-oatao@listes-diff.inp-toulouse.fr 


\title{
A High Voltage High Frequency Resonant Inverter for Supplying DBD Devices with Short Discharge Current Pulses
}

\author{
Xavier Bonnin, Julio Brandelero, Nicolas Videau, Hubert Piquet, and Thierry Meynard
}

\begin{abstract}
In this paper, the merits of a high-frequency resonant converter for supplying dielectric barrier discharges (DBD) devices are established. It is shown that, thanks to its high-frequency operating condition, such a converter allows to supply DBD devices with short discharge current pulses, a high repetition rate, and to control the injected power. In addition, such a topology eliminates the matter of connecting a high-voltage transformer directly across the DBD device and avoids the issues related to the parasitic capacitances of the latter which disturbs the control the power transfer to the plasma. The design issues of the converter, including the inverter and its switches, the resonant inductor, and the parameter drift compensation are studied. An experimental validation is performed: a mega Hertz resonant converter using GaN FET switches has been manufactured and tested with an excimer lamp.
\end{abstract}

Index Terms-Dielectric barrier discharges (DBD), GaN, high frequency, high voltage, inverter, mega-hertz, plasma, pulsed power, resonance, transformerless, wide bandgap.

\section{INTRODUCTION}

D IELECTRIC barrier discharges (DBDs) are nowadays extensively used to achieve the generation of cold plasmas at atmospheric pressure [1], [2]. Industrial applications of DBDs are spread out to various industrial domains, such as surface treatment [3]-[5], decontamination [6], [7], semiconductor fabrication, lighting — especially UV rays generation [8] by means of DBD excimer lamps.

In order to supply such a capacitive load, several options using resonant or quasi-resonant principles have been studied in [9]-[11]. In [9] and [10], topologies using a high-voltage transformer are used in order to deal with the high voltage necessary to supply the DBD device. A low-frequency transformerless topology using the voltage gain of an LCL cell at a resonant frequency is studied in [11]. The operating frequencies are in the range of $30 \mathrm{kHz}$ in [9] and [11] and of $80 \mathrm{kHz}$ in [10].

X. Bonnin, N. Videau, H. Piquet, and T. Meynard are with the LAPLACE (Laboratoire Plasma et Conversion d'Énergie), Université de Toulouse 31071 Toulouse cedex 7, France (e-mail: xavier.bonnin@ laplace.univ-tlse.fr; videau@ laplace.univ-tlse.fr; piquet@laplace.univ-tlse.fr; meynard@laplace.univ-tlse. fr).

J. Brandelero is with the CIRTEM company, Labège 31670, France (e-mail: brandelero@laplace.univ-tlse.fr).
Already few hundreds of kilo-Hertz frequency range has been reached [12] by using a current-controlled inverter associated with a high-voltage transformer directly connected to the DBD device.

Topologies using a high-voltage transformer offer design eases by strongly reducing the working voltage at the primary side and strongly increasing the equivalent capacitance seen at the primary winding. It is shown that including a high-voltage transformer may lead to capacitive effects harmful for the proper functioning of the system [13]. Capacitive effects, which can be taken into account by means of a single capacitance connected in parallel with the DBD device, forms a current divider: an important part of the supplied current flows through the equivalent parasitic capacitance, instead of the DBD device, complicating the gas ignition and leading to increase the transformer current rating.

In some industrial applications, like surface treatment and lighting, pulsed discharge currents have proved their interest [14], [15]. Many pulsed power converters designed for DBD applications have already been considered. In [16], a Marx generator-based topology allows us to obtain few hundreds of nanoseconds pulsed discharges and a repetition rate about several hundreds of Hertz. In [17], a voltage inverter topology is implemented, and few microseconds pulses durations is obtained with a repetition rate of few tens of kilohertz. Such topologies do not allow us to reach high pulse repetition rates. In [17], the use of a high voltage transformer directly connected to the DBD device also exhibits a lower limit value for the pulses durations because of the leakage inductance.

The purpose of this paper is to show that a high-frequency resonant topology allows us to supply DBD devices with controlled short discharge currents with high repetition rates. It is also shown that short-current pulses durations can be obtained because no transformer directly connected to the DBD device is needed. High-frequency (above $1 \mathrm{MHz}$ ) resonant converters have already been studied for high current / medium voltage discharge lamps in [18].

In Section II, the DBD device behavior is described, and a theoretical study is carried out in order to establish the merits of such a power converter: it is shown that it is possible to control the injected power and the discharge pulses duration using the power converter parameters. Then, in Section III, the design issues of the resonant converter including the resonant inductor and the inverter are considered in order to formulate the specifications. Finally, Section IV brings an experimental 


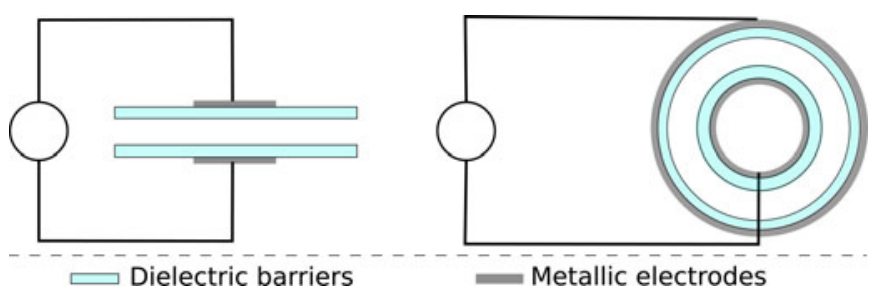

Fig. 1. Two types of DBD: planar and cylindrical geometries.

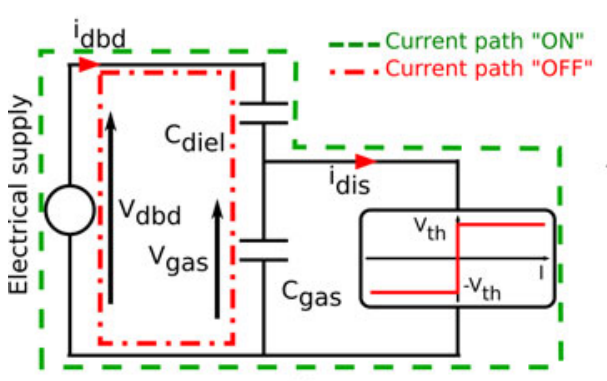

(a)

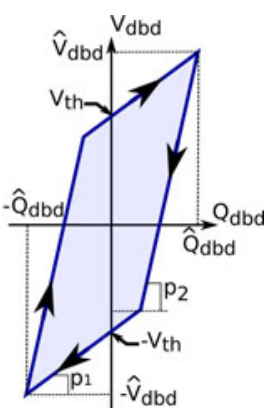

(b)

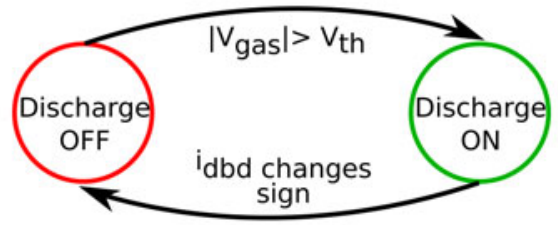

(c)

Fig. 2. (a) Electrical model of a DBD device. (b) Charge-potential diagram under periodic operating conditions. (c) Sequential diagram for the operating sequences.

validation which exhibits technical limitations and highlights the challenge of controlling parasitic elements.

\section{THEORETICAL STUDY}

\section{A. DBD Devices}

1) Characteristics: Two examples of DBD devices are represented in Fig. 1. The first example shows an experimental setup made of two parallel plates which is commonly used to carry out experimental validations for surface treatment applications. The second one shows a cylindrical setup which is commonly used in UV-emission devices.

2) Electrical Behavior: According to [19], such a device can be electrically modeled by the equivalent scheme; see Fig. 2(a). It sufficiently describes both cylindrical and planes geometries setups. $C_{\text {gas }}$ models the structural capacitance of the gas volume when the discharge is not alighted, $C_{\text {diel }}$ stands for the total dielectric capacitance due to the presence of at least one dielectric barrier. $V_{\mathrm{th}}$ the steady-state threshold voltage, models the breakdown phenomenon that occurs when the gas voltage $V_{\text {gas }}$ is increased. In some DBD devices like excimer lamps, the first gas ignition threshold voltage $V_{\text {th }}^{[1]}$ is greater than the steady-state threshold voltage $V_{\text {th }}$. For a "worst case" design
TABLE I

Typical Parameter Values of Two DBD Devices

\begin{tabular}{lcr}
\hline & $\begin{array}{c}\text { Planar DBD } \\
\left(9 \mathrm{~cm}^{2} / 1 \mathrm{~mm} \text { gap }\right)\end{array}$ & Excimer lamp \\
\hline Application & $\begin{array}{c}\text { Surf. treatment } \\
\text { (experimental setup) }\end{array}$ & UV emission \\
& Planar & Cylindrical \\
Geometry type & $50 \mathrm{~W}$ & $100 \mathrm{~W}$ \\
Power (typical) & $0.1-15 \mathrm{kHz}$ & $50-200 \mathrm{kHz}$ \\
Frequency range & $50-150$ & $10-20$ \\
HV transformer ratio & $12 \mathrm{kV}$ & $5 \mathrm{kV}$ \\
Max. voltage & $50 \mathrm{~mA}$ & $50 \mathrm{~mA}$ \\
Typ. current & $8 \mathrm{pF}$ & $21 \mathrm{pF}$ \\
$C_{\text {gas }}$ & $55 \mathrm{pF}$ & $75 \mathrm{pF}$ \\
$C_{\text {diel }}$ & $3500 \mathrm{~V}$ & $1200 \mathrm{~V}$ \\
$V_{\text {th }}$ & & \\
\hline
\end{tabular}

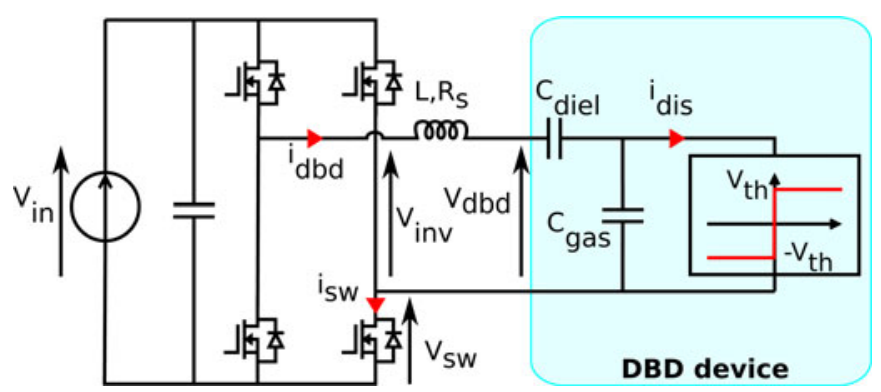

Fig. 3. Resonant converter topology: outline schematic. $R_{s}$ stands for modeling losses elements.

$V_{\mathrm{th}}^{[1]}$ should be employed instead of $V_{\mathrm{th}}$. The latter would be employed for steady-state calculations. DBD devices must be supplied with ac current sources because of the presence of the dielectric barriers. A common approach which facilitates the reasoning under periodic operating condition is to model the DBD device by its $Q_{\mathrm{dbd}}-V_{\mathrm{dbd}}$ diagram, where $Q_{\mathrm{dbd}}$ and $V_{\mathrm{dbd}}$ are, respectively, the injected charge into the DBD device defined by $Q_{\mathrm{dbd}}=\int i_{\mathrm{dbd}}$ and the DBD voltage. It leads to the results shown in Fig. 2(b). The slopes $p_{1}$ and $p_{2}$ stand, respectively, for $1 / C_{\text {diel }}$ and $1 / C_{\text {diel }}+1 / C_{\text {gas }}$.

$E_{\mathrm{dbd}}^{T}$ the energy injected into the DBD device during one period is defined by

$$
E_{\mathrm{dbd}}^{T}=\int_{T} V_{\mathrm{dbd}} i_{\mathrm{dbd}} d t=\oint V_{\mathrm{dbd}} d Q_{\mathrm{dbd}}=\mathcal{A} .
$$

The value of $E_{\mathrm{dbd}}^{T}$ is $\mathcal{A}$, the area of the parallelogram [see Fig. 2(b)]. Typical values of DBD devices parameters used for our experiments are displayed in Table I. The same values are observed in [10] and [20]. Capacitance values are typically of several tens of picoFarad, and the threshold voltage $V_{\mathrm{th}}$ is around several kiloVolts.

\section{B. Theoretical Study}

1) Converter Topology: Because of their high threshold voltage value, supplying DBD devices needs to deliver high voltage in spite of the small values of $C_{\mathrm{gas}}$ and $C_{\mathrm{diel}}$. A resonant topology described in Fig. 3 is appropriate in that regard. Indeed, high-voltage values across the DBD can be reached thanks to the resonant tank voltage gain. It consists of a low-voltage source $V_{\text {in }}$, whose value is compatible with common power electronics 
switches, connected to a full-bridge inverter which supplies with a symmetrical squared voltage to the resonant tank, made of the resonant inductance connected in series with the DBD device.

2) Injected Power Under Periodic Operating Condition: In this section, the effects of $R_{s}$, which account for the losses of the bridge and the inductor, are neglected. On the one hand, the mean power consumed by the DBD device $P_{\mathrm{dbd}}$ is given by the common formula

$$
P_{\mathrm{dbd}}=\frac{1}{T} \int_{0}^{T} i_{\mathrm{dbd}}(t) V_{\mathrm{inv}}(t) d t
$$

where $T$ is the operating period. The current $i_{\mathrm{dbd}}$ is assumed to be quasi-sinusoidal with a frequency $F$ because of the resonant tank which achieves a low-pass filtering and eliminates the harmonics of $V_{\text {inv }}$. Thus, (2) leads to

$$
\begin{aligned}
P_{\mathrm{dbd}}= & \frac{1}{T} \int_{0}^{T} \hat{i}_{\mathrm{dbd}} \sin (2 \pi F t) \\
& \times \sum_{n=1}^{\infty} \hat{V}_{\mathrm{inv}}^{(n)} \sin \left(2 \pi n F t+\phi_{n}\right) d t \\
= & \frac{1}{2} \hat{V}_{\mathrm{inv}}^{(1)} \hat{i}_{\mathrm{dbd}} \cos \left(\phi_{1}\right) \\
= & \pi F \hat{V}_{\mathrm{inv}}^{(1)} \hat{Q}_{\mathrm{dbd}} \cos \left(\phi_{1}\right)
\end{aligned}
$$

where $\hat{i}_{\mathrm{dbd}}, \hat{Q}_{\mathrm{dbd}}$, and $\hat{V}_{\text {inv }}^{(1)}$ are, respectively, the magnitudes of $i_{\mathrm{dbd}}, Q_{\mathrm{dbd}}$, and the fundamental of $V_{\mathrm{inv}}$, and $\phi_{1}$ is the phase difference between the first harmonics of $i_{\mathrm{dbd}}$ and $V_{\mathrm{inv}}$.

On the other hand, the energy injected into the DBD device during one period is given by the $Q-V$ diagram area, $\mathcal{A}$. A geometrical study of Fig. 2(b) shows that

$$
\mathcal{A}=4 V_{\mathrm{th}}\left(\hat{Q}_{\mathrm{dbd}}-C_{\mathrm{gas}} V_{\mathrm{th}}\right) .
$$

The mean power consumed by the DBD device is given by $P_{\mathrm{dbd}}=F \mathcal{A}$. Thus, combining (3) and (4) leads to

$$
\hat{Q}_{\mathrm{dbd}}=\frac{C_{\mathrm{gas}} V_{\mathrm{th}}}{1-\frac{\pi \hat{V}_{\mathrm{inv}}^{(1)}}{4 V_{\mathrm{th}}} \cos \left(\phi_{1}\right)} .
$$

Therefore, we get

$$
P_{\mathrm{dbd}}=\frac{\pi F C_{\mathrm{gas}} V_{\mathrm{th}} \hat{V}_{\mathrm{inv}}^{(1)} \cos \left(\phi_{1}\right)}{1-\frac{\pi \hat{V}_{\mathrm{inv}}^{(1)}}{4 V_{\mathrm{th}}} \cos \left(\phi_{1}\right)}
$$

by combining (3) and (5). Moreover, because a high voltage gain is expected for the resonant tank, it is assumed that $\hat{V}_{\text {inv }}^{(1)} \ll V_{\text {th }}$, leads to

$$
P_{\mathrm{dbd}} \approx \pi F C_{\mathrm{gas}} V_{\mathrm{th}} \hat{V}_{\mathrm{inv}}^{(1)} \cos \left(\phi_{1}\right) .
$$

Expected waveforms taken from a SPICE simulation are plotted in Fig. 4. In a resonant converter, $\phi_{1}$ depends on $F$. Furthermore, because of the expected resonant tank high selectivity, $F$ can be assumed to be nearly constant, and $\phi_{1}$ is a degree of freedom allowing to control the injected power. Therefore, (7) shows that $P_{\mathrm{dbd}}$ can be controlled by either controlling $\phi_{1}$ by acting very slightly on $F$, or by acting on $\hat{V}_{\mathrm{inv}}^{(1)}$. The later can be performed by either acting on $V_{\text {in }}$ when it is technically possible or by

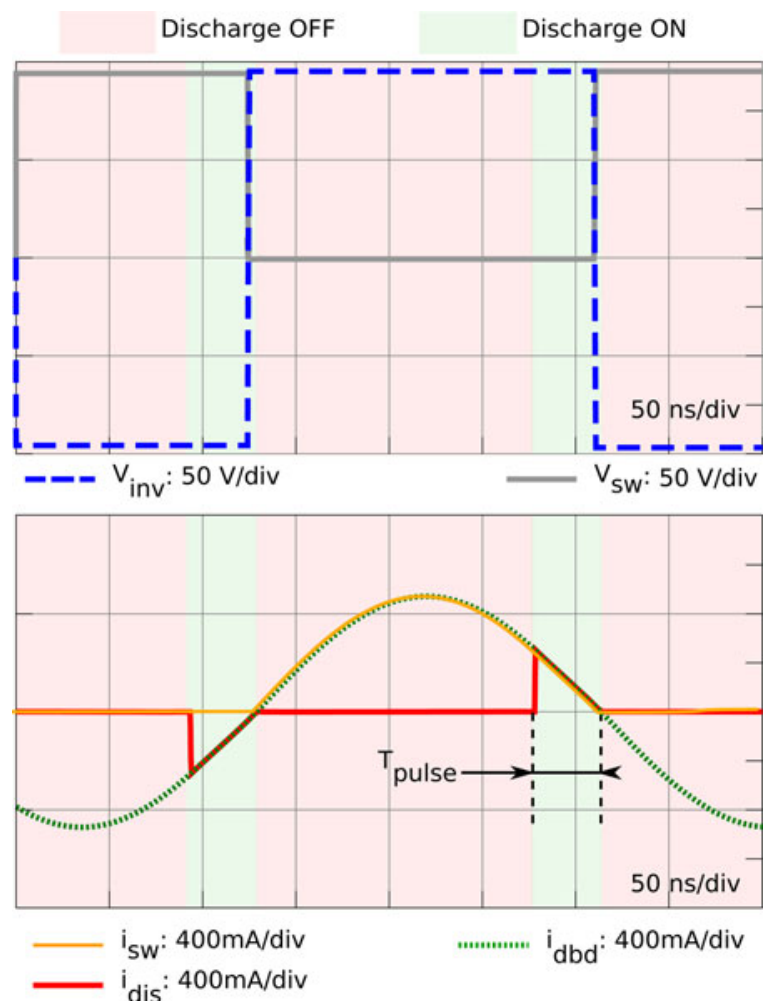

Fig. 4. Spice simulation results: expected waveforms with $V_{\text {in }}=100 \mathrm{~V}$ $C_{\mathrm{gas}}=21 \mathrm{pF}, C_{\mathrm{diel}}=75 \mathrm{pF}, V_{\mathrm{th}}=1200 \mathrm{~V}, L=205 \mu \mathrm{H}, R_{s}=0 \Omega F=$ $2.708 \mathrm{MHz}$. Both theoretical study and computation give $P_{\mathrm{dbd}} \approx 29 \mathrm{~W}$ and $T_{\text {pulse }} \approx 36 \mathrm{~ns}$.

acting on the switches command law. In addition, the relative drift of $P_{\mathrm{dbd}}$ equals the relative drift of the physical parameters in the worst case since (7) leads to

$$
\left|\frac{d P_{\mathrm{dbd}}}{P_{\mathrm{dbd}}}\right|_{\phi_{1}, \hat{V}_{\mathrm{inv}}^{(1)}} \leq\left|\frac{d V_{\mathrm{th}}}{V_{\mathrm{th}}}\right|+\left|\frac{d C_{\mathrm{gas}}}{C_{\mathrm{gas}}}\right|
$$

3) Pulses Geometry: $P_{\mathrm{dbd}}$ can be calculated by distinguishing the discharge ON and OFF states. Effective power is transferred only during the ON states. Equation (2) leads to

$$
P_{\mathrm{dbd}}=2 F \int_{\text {pulsewidth }} V_{\mathrm{th}} i_{\mathrm{dbd}}(t) d t .
$$

Moreover, the discharge transits from the ON state to the OFF state when $i_{\mathrm{dbd}}$ changes the sign. Therefore, assuming that $i_{\mathrm{dbd}}(t)=\hat{i}_{\mathrm{dbd}} \sin (2 \pi F t)$ because of the resonant tank high selectivity, leads to

$$
\begin{aligned}
P_{\mathrm{dbd}} & =V_{\mathrm{th}} \frac{\hat{i}_{\mathrm{dbd}}\left(1-\cos \left(2 \pi F T_{\text {pulse }}\right)\right)}{\pi} \\
& =2 F V_{\mathrm{th}} \hat{Q}_{\mathrm{dbd}}\left(1-\cos \left(2 \pi F T_{\text {pulse }}\right)\right) \\
& \left.=4 F V_{\mathrm{th}} \hat{Q}_{\mathrm{dbd}} \sin ^{2}\left(\pi F T_{\text {pulse }}\right)\right)
\end{aligned}
$$




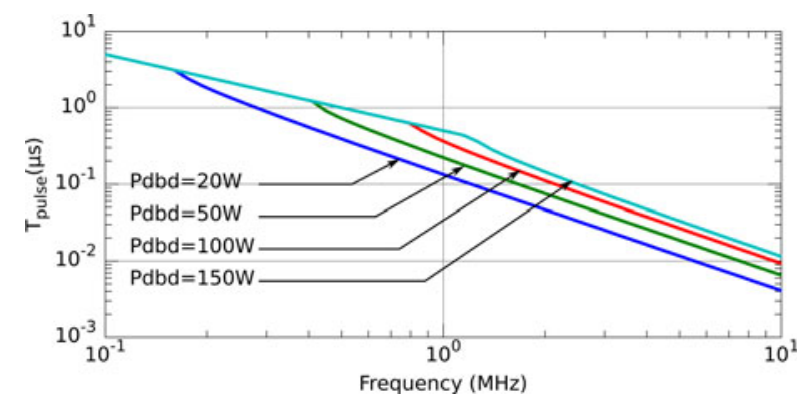

Fig. 5. $T_{\text {pulse }}$ as a function of the operating frequency for $V_{\mathrm{th}}=1200 \mathrm{~V}$ and $C_{\mathrm{gas}}=21 \mathrm{pF}$.

where $T_{\text {pulse }}$ is the pulse duration. Combining (5) and (10) leads to

$$
\begin{aligned}
T_{\text {pulse }} & =\frac{1}{\pi F} \arcsin \sqrt{\frac{P_{\mathrm{dbd}}\left(1-\frac{\pi \hat{V}_{\mathrm{inv}}^{(1)} \cos \left(\phi_{1}\right)}{4 V_{\mathrm{th}}}\right)}{4 F C_{\mathrm{gas}} V_{\mathrm{th}}^{2}}} \\
& \approx \frac{1}{\pi F} \arcsin \sqrt{\frac{P_{\mathrm{dbd}}}{4 F C_{\mathrm{gas}} V_{\mathrm{th}}^{2}} .}
\end{aligned}
$$

The theoretical value of $T_{\text {pulse }}$ is plotted in Fig. 5 .

The high frequency range $(1-10 \mathrm{MHz})$ interest is demonstrated since it allows us to reach low-pulse durations (10$100 \mathrm{~ns})$.

\section{Design Issue OF THE RESONANT POWER CONVERTER}

\section{A. Resonant Converter and Its Losses}

1) Voltage Gain of the Resonant Tank: Actually, the power converter elements are subject to losses which, in addition to reducing the converter efficiency, also can reduce significantly the resonant tank voltage gain. This may prevent the discharge from igniting. In this section, losses are encompassed in a serial resistor $R_{s}$ (see Fig. 3) with the resonant inductance assumed to be a constant parameter at the working frequency.

In order to ignite the discharge, $V_{\text {gas }}$ has to reach $V_{\mathrm{th}}^{[1]}$. Consequently, the voltage gain $G_{v}$ of the converter defined as the the ratio $\hat{V}_{\mathrm{dbd}} / \hat{V}_{\text {inv }}^{(1)}$ must fulfill the following criterion:

$$
G_{v}>\left(1+\frac{C_{\mathrm{gas}}}{C_{\mathrm{diel}}}\right) \frac{V_{\mathrm{th}}^{[1]}}{\hat{V}_{\mathrm{inv}}^{(1)}}
$$

Moreover, taking into account that $G_{v} \gg 1$ because a high voltage gain is expected for the resonant tank, it can be approximated by the classical formula for serial $R L C$ resonant circuits:

$$
G_{v}=Q=\frac{1}{R_{s}} \sqrt{\frac{L}{C_{\mathrm{eq}}}}
$$

where $C_{\mathrm{eq}}=C_{\mathrm{gas}} C_{\mathrm{diel}} /\left(C_{\mathrm{gas}}+C_{\mathrm{diel}}\right)$ and $Q$ are respectively the "OFF state" resonant capacitance and the load quality factor. Thus, introducing the "OFF state" resonant pulsation $\omega_{0}=1 /\left(\sqrt{L C_{\text {eq }}}\right)$ of the load in (13) leads to

$$
G_{v}=\frac{L \omega_{0}}{R_{s}}
$$

Finally, the criterion (12) is expressed as

$$
\frac{L \omega_{0}}{R_{s}}>\left(1+\frac{C_{\mathrm{gas}}}{C_{\mathrm{diel}}}\right) \frac{V_{\mathrm{th}}^{[1]}}{\hat{V}_{\mathrm{inv}}^{(1)}} .
$$

Criterion (15) intends to provide guidance concerning the design of the resonant inductor and the switches of the full-bridge. Therefore, the challenge is to control both the value of $L$ in order to reach a desired resonant frequency and the value of $R_{s}$ in order to satisfy (15).

2) Switching Conditions: Contrary to power converters that do not use the resonant principle for supplying DBD devices, good switching conditions can be obtained and switching losses can be reduced. Fig. 4 shows that when $V_{\text {inv }}$ changes of sign, the load current $i_{\mathrm{dbd}}$ is nearly zero meaning that a ZVS and ZCS behavior can be obtained.

\section{B. Designing the Resonant Inductor and the Inverter}

Losses are located mainly in the magnetic core (hysteresis and eddy current losses) and in the winding structure (dc resistance, skin, and proximity effects). For a given frequency, the total losses can be modeled by a single serial resistance $R_{s}$.

1) Magnetic Material Survey: Iron losses contribute at increasing $R_{s}$ value: a wise choice of the magnetic material is important. Usually, manufacturers give either fitting coefficients $K, \alpha, \beta$ for the Steinmetz equation giving the loss density $p_{\text {tot }}$ as $p_{\text {tot }} \approx K \hat{B}^{\alpha} F^{\beta}$ or either the complex permeability $\underline{\mu}=\mu^{\prime}-j \mu^{\prime \prime}$ where $\mu^{\prime}$ and $\mu^{\prime \prime}$ are positive values, as a function of the operating frequency. The first approach furnishes a behavioral law that takes into account both hysteresis and $e d d y$ current losses for a sinusoidal induction although the second one serves at evaluating hysteresis loss density $p_{\text {hyst }}$. The hysteresis loss factor $\tan \delta_{\text {hyst }}$ of the material is defined as

$$
\tan \left(\delta_{\text {hyst }}\right)=\frac{\mu^{\prime \prime}}{\mu^{\prime}}=\frac{R_{s}^{\text {hyst }}}{L \omega}
$$

where $\omega=2 \pi F, F$ being the working frequency, and $R_{s}^{\text {hyst }}$ is the equivalent serial resistance with the inductance due to total hysteresis losses. Assuming that $\delta \ll 1$ and for a sinusoidal excitation, the hysteresis loss density can be estimated by

$$
p_{\text {hyst }} \approx \frac{\omega \tan \left(\delta_{\text {hyst }}\right)}{2|\underline{\mu}|} \hat{B}^{2} .
$$

Analogously, a total loss factor $\tan \left(\delta_{\text {tot }}\right)$ can be defined. As $\tan \left(\delta_{\text {hyst }}\right)$ fulfills $(17), \tan \left(\delta_{\text {tot }}\right)$ should be such as

$$
p_{\text {tot }}=\frac{\omega \tan \left(\delta_{\text {tot }}\right)}{2|\underline{\mu}|} \hat{B}^{2}
$$

leading to

$$
\begin{aligned}
\tan \left(\delta_{\mathrm{tot}}\right) & \approx \frac{2|\underline{\mu}| p_{\mathrm{tot}}}{\omega \hat{B}^{2}} \\
& \approx \frac{|\underline{\mu}| K \hat{B}^{\alpha-2} F^{\beta-1}}{\pi}
\end{aligned}
$$

by using the behavioral law of $p_{\text {tot }}$. By analogy with (16), the equivalent serial resistance responsible for the inductance core 


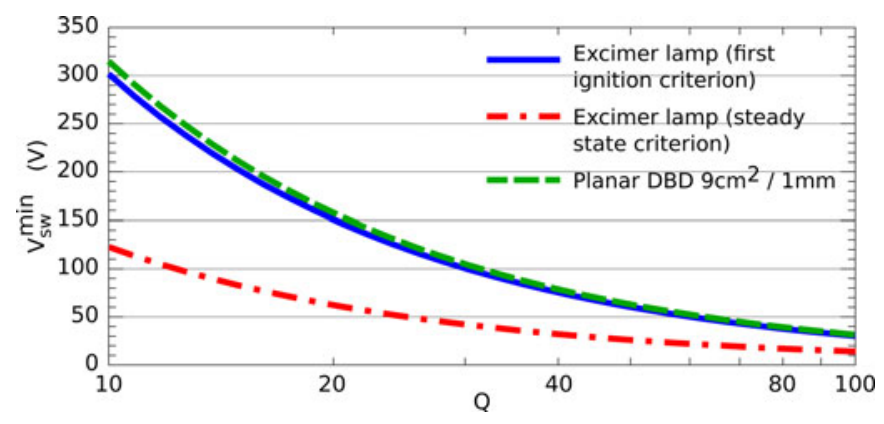

Fig. 6. Switches voltage rating as a function of the quality factor. Numerical values are taken from Table I. It is experimentally observed that $V_{\mathrm{th}}^{[1]} \approx 3000$ $\mathrm{V}$ for the excimer lamp, and $3500 \mathrm{~V}$ for the plate/plate device.

total losses $R_{s}^{\text {tot }}$ such as

$$
\tan \left(\delta_{\mathrm{tot}}\right)=\frac{R_{s}^{\mathrm{tot}}}{L \omega} .
$$

can be defined. Thus, according to (20), criterion (15) exhibits an upper limit to the magnetic material loss factor that can be expressed as

$$
\tan \left(\delta_{\mathrm{tot}}\right)<\frac{1}{\left(1+\frac{C_{\mathrm{gas}}}{C_{\mathrm{diel}}}\right)} \frac{\hat{V}_{\mathrm{inv}}^{(1)}}{V_{\mathrm{th}}^{[1]}} .
$$

2) Winding Arrangements: The resonant inductance is designed to produce an high voltage across the DBD device. Therefore, it has to withstand a high voltage since its voltage is $V_{\mathrm{dbd}} \pm V_{\text {in }}$ which can reach several kilovolts. Therefore, in order to prevent from arcing, the turns have to be arranged in order to be far apart from each other, and a highly isolated core have to be used.

\section{3) Inverter Switches:}

a) Switches voltage rating: The criterion (12) can be rewritten as

$$
\hat{V}_{\text {inv }}^{(1)}>\frac{\left(1+\frac{C_{\text {gas }}}{C_{\mathrm{diel}}}\right) V_{\mathrm{th}}^{[1]}}{Q} .
$$

In the case of a symmetric square voltage waveform of magnitude $V_{\text {in }}$, the Fourier expansion allows us to express the first harmonic magnitude by $\hat{V}_{\text {inv }}^{(1)}=4 / \pi V_{\text {in }}$. It leads to express the switches minimum voltage rating $V_{\mathrm{sw}}^{\mathrm{min}}$ as

$$
V_{\mathrm{sw}}^{\min }=\frac{\pi\left(1+\frac{C_{\mathrm{gas}}}{C_{\mathrm{diel}}}\right) V_{\mathrm{th}}^{[1]}}{4 Q} .
$$

Typical $V_{\mathrm{sw}}^{\min }$ versus $Q$ curves are sketched in Fig. 6.

Two cases are taken into account: the "first ignition criterion" since the power converter switches have to carry a higher voltage to ignite the gas the first time, and the "steady state criterion" which assumes that an auxiliary circuit is in charge of the first gas ignition. Fig. 6 shows that, if $Q$ is high enough, the gas ignition can be reached with common electronic switches.

b) Switches losses: Conduction losses $P_{\text {cond }}$ can be modeled by $P_{\text {cond }}=R_{\mathrm{ds}} i_{\mathrm{rms}}^{2}$, where $R_{\mathrm{ds}}$ stands for the on-state drain-source resistance, and $i_{\mathrm{rms}}$ is the RMS switch current. In addition, switching losses can be modeled by considering

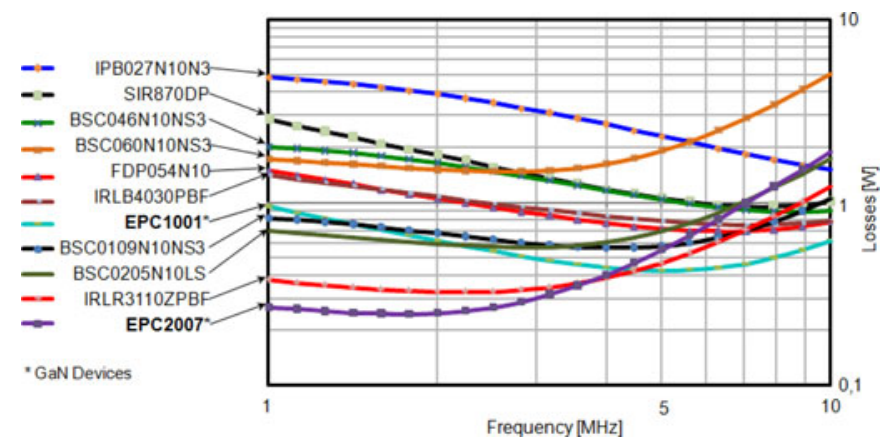

Fig. 7. Switch losses versus frequency for several switches having a voltage rating of $100 \mathrm{~V}$. The parameters $V_{\text {in }}$ and $i_{\mathrm{rms}}$ change with the frequency value to ensure a constant power of $66 \mathrm{~W}$. All the components are MOSFET devices except the EPC1001 and EPC2007 which are GaN devices.

the switching sequence, including the transistors dead time. For this study, it is assumed that the switching time is much shorter than the operating period, and that during the switching time, the DBD current is zero (soft-switching assumption which is experimentally observed in Fig. 16). According to [21], 0-A switching losses $P_{\mathrm{sw}}$ are only due to the charge/discharge of the switch output parasitic capacitance $C_{\text {oss }}$ wose value depends on the drain-source voltage. Thus, the switching losses are given by $P_{\mathrm{sw}}=F \int_{0}^{V_{\text {in }}} C_{\mathrm{oss}} V_{\mathrm{ds}} d V_{\mathrm{ds}}$, where $F$ is the switching frequency, and $V_{\text {in }}$ is the input voltage. According to [22], $C_{\text {oss }}$ can be expressed as $C_{\mathrm{oss}}=C_{0} /\left(1+V_{\mathrm{ds}} / K\right)^{\gamma}$ for MOSFET devices, where $C_{0}$ is the zero-voltage capacitance and $K, \gamma$ are adjustments parameters. Therefore, switch losses are given by

$$
P_{\mathrm{tot}}=P_{\text {cond }}+P_{\mathrm{sw}}=R_{\mathrm{ds}} i_{\mathrm{rms}}^{2}+F \int_{0}^{V_{\mathrm{in}}} C_{\mathrm{oss}} V_{\mathrm{ds}} d V_{\mathrm{ds}} .
$$

In order to provide guidance in the choice of the power switches, a comparison of several switches having the lowest figures of merit $\left(Q_{g} \times R_{d s}\right.$ product) is given in Fig. 7. The comparison is done at a constant output power $P_{\mathrm{dbd}}=66 \mathrm{~W}$. Equation (7) shows that the DBD power $P_{\mathrm{dbd}}$ scales in $V_{\text {in }} \times F$. Therefore, the input voltage $V_{\text {in }}$ must scale in $1 / F$ in order to ensure a constant power in the DBD device. Moreover, $i_{\mathrm{rms}}$ must scale in $F$ because the input power scales in $V_{\text {in }} \times i_{\text {rms }}$. Fig. 7 shows that, for this application, devices made of $\mathrm{GaN}$ material are serious candidates among the best state of art MOSFET devices. Further improvements can be expected since GaN devices are far from their theoretical limitation in term of specific resistance and breakdown voltage [23].

Because of the high switching frequency, a short switching time (few nanoseconds) is recommended. Both parasitic elements of the switches [24] and PCB layout play a determining role in that regard. In [25], the authors show how the PCB layout can affect a fast switching high-frequency converter efficiency, and how an optimized layout can be performed in order to prevent from switches voltage overshoots and to reduce switching losses.

4) Parameters Drift Compensation: Physical parameters: $V_{\mathrm{th}}, C_{\mathrm{gas}}$ for the DBD device and $\mu$ for the resonant inductor, are subjected to fluctuations. Fluctuations can be either slow, due 


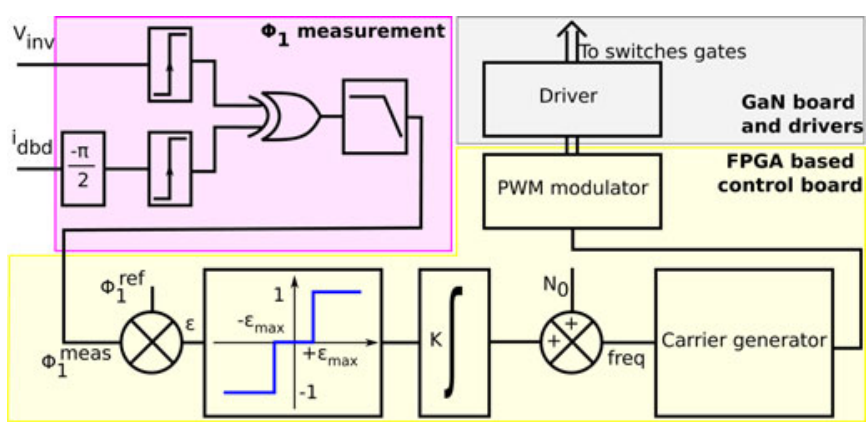

Fig. 8. Operating frequency control to compensate the parameters fluctuation.

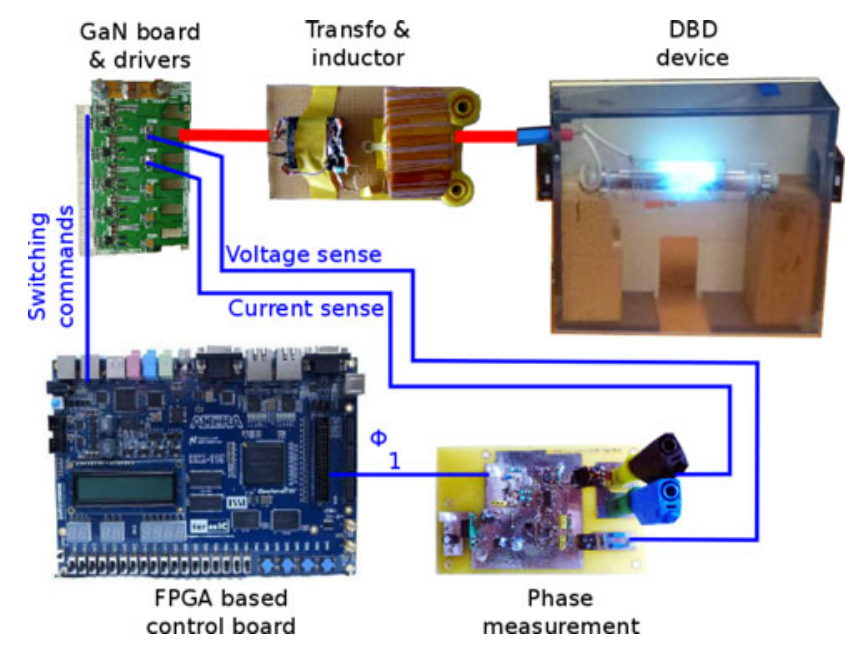

Fig. 9. Outline of the experimental setup.

to temperature variations, or abrupt, due to a component value change. These variations affect the load resonant frequency, and strongly impact the injected power since $\phi_{1}$ is very sensitive to frequency fluctuations. A control system suitable for electric discharge application is studied in [26]. It allows us to compensate abrupt and slow parameters variations and ensures a better discharge control. Our implementation compensates only slow variations (thermal drifts) by means of controlling the phase difference between $V_{\mathrm{inv}}$ and $i_{\mathrm{dbd}}$ in order to maintain the system at the resonant frequency. A scheme of the operating frequency control is presented in Fig. 8. The phase difference between $i_{\mathrm{dbd}}$ and $\hat{V}_{\mathrm{inv}}^{(1)}$ is measured with a phase comparator, and is compared with the phase reference $\phi_{1}^{\text {ref }}$. Then, the carrier generator frequency is controlled by a control block which ensures a zero static error.

\section{EXPERIMENTAL VALIDATION}

The experimental setup is presented in Fig. 9.

\section{A. Experimental Setup}

1) Inverter: The inverter used for the experiment is taken from a $\mathrm{GaN}$ high-frequency converter whose design is studied in [27]. To reach the selected frequency range $(1 \mathrm{MHz})$, the PCB layout and the components placement are performed in order to minimize the switching losses and to prevent from

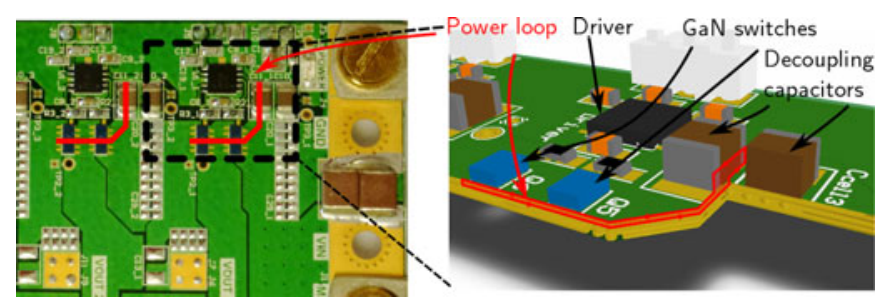

Fig. 10. Zoom on the GaN switched inverter two cells. Power loop surface is minimized.

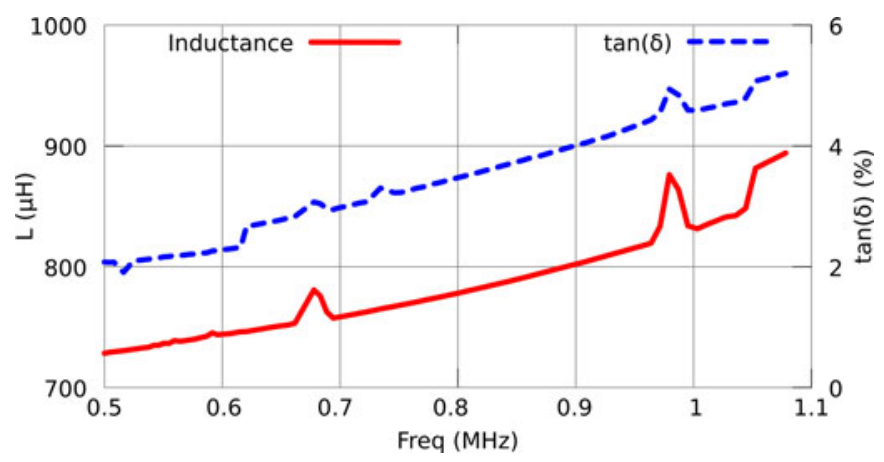

Fig. 11. Actual resonant inductor: values of $L$ and of $\tan (\delta)$ versus frequency. Measurements are performed with the Newton N4L impedance-meter.

GaN voltage overshoots: the power decoupling capacitors placement, being on the same side of the board as the GaN switches, both minimize the commutation cell stray inductances and facilitates a heatsink implementation (see Fig. 10). As shown in Fig. 10, care is taken about minimizing switching cells power path surfaces. The switches are $100-\mathrm{V}$ nitride gallium eGaN FET EPC1001 [28].

2) Resonant Inductor: The resonant inductor is made of a nanocrystalline material $k_{\mu} 200$ toroid core KJ030020Y200 [29] and of 60 turns of $1 \mathrm{~mm} / 100$ strands circular Litz wire. Measured characteristics are plotted in Fig. 11. At $1 \mathrm{MHz}$, the measured inductance value is $820 \mu \mathrm{H}$ and the loss factor, which includes both iron and copper losses, is close to 0.047 leading to $R_{s} \approx 240 \Omega$. Therefore, according to (13), the expected voltage gain is about 21. Moreover, this resonant inductor exhibits an equivalent parallel parasitic capacitance of $6 \mathrm{pF}$ which may bring out a parasitic resonance peak (see the study in Section IV-A3).

3) Medium-Voltage Transformer: Because of the inverter voltage rating $(60 \mathrm{~V})$ and the inductor performance $(\tan (\delta)=$ $4.7 \%$, so $Q=21.2$ ), the maximum DBD voltage that can be reached may be not adequate to reach the first discharge ignition (see Fig. 6). In Fig. 12, the lamp is not ignited although its voltage amplitude is $3.5 \mathrm{kV}$. However, once the first ignition has been reached, the lamp ignition voltage decreases. For this reason, a medium-voltage high-frequency transformer is inserted between the the inverter and the inductance. It consists of an EFD 25/13/9-3F4 magnetic core (Ferroxcube). The turns are disposed in one single layer and a large insulation layer separates the primary and the secondary windings in order to minimize the capacitive effects. Its turn ratio is 4 in order to provide an ac voltage in the $0-240 \mathrm{~V}$ range, and to explore a large 


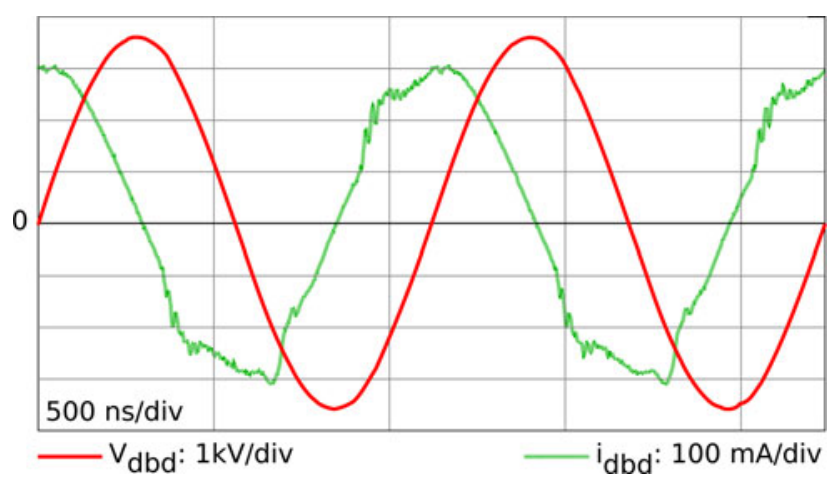

Fig. 12. Experimental waveforms obtained before the first ignition of the lamp.

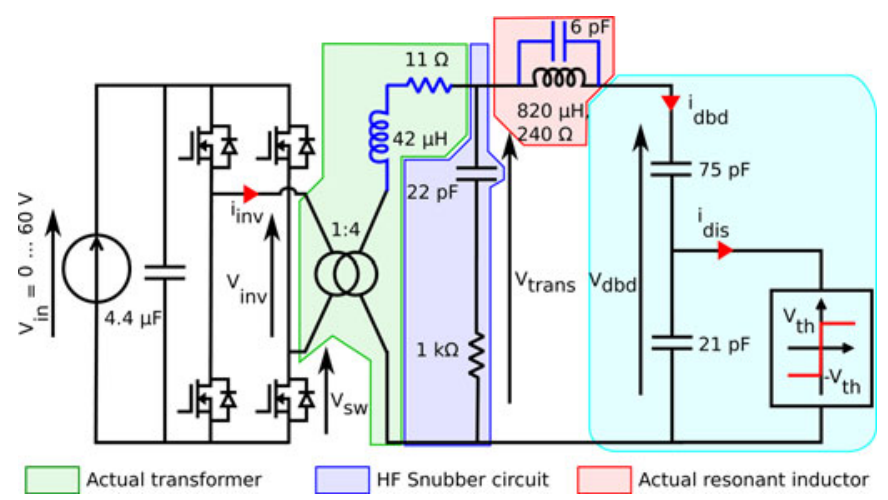

Fig. 13. Electrical scheme of the actual experimental setup with the medium voltage transformer. Only the most significant elements are taken into account.

DBD operating point range. Theoretically, with the transformer, $6500 \mathrm{~V}$ can be obtained before the first ignition.

When the transformer is placed upstream the resonant inductor, the capacitive leakage current matter evoked in [13] is avoided. According to [30], under the assumptions that the transformer is highly coupled and that the leakage inductance voltage drop can be neglected, the transformer capacitive effects can be approximated with a single capacitance $C_{p}$ connected across the secondary winding. The $C_{p}$ value depends on the transformer building parameters and the way that it is grounded. The $C_{p}$ value is typically in the range of several tens of picofarads for the transformers used for our experiments. The leakage current matter appears when the DBD device is directly connected to the secondary winding because of the low impedance ratio $\left|Z_{C_{p}} / Z_{\mathrm{DBD}}\right|$, where $Z_{\mathrm{DBD}}$, essentially made of a capacitive term, stands for the DBD device impedance which depends on the gas state (ignited or not). It means that an important amount of current flows into $C_{p}$ instead of the DBD device. However, when the resonant inductor is placed on the secondary side of the transformer, the impedance ratio $\left|Z_{C_{p}}\right| Z_{\text {load }} \mid$, where $Z_{\text {load }}=Z_{\mathrm{DBD}}+j L \omega$, is much higher since the $j L \omega$ term compensates the capacitive term of $Z_{\mathrm{DBD}}$ at the resonant frequency. Nevertheless, the circuit frequency response is affected by the leakage inductance of the transformer. Fig. 13 shows the electrical scheme of the experimental setup that takes into account the most significant elements. In Fig. 14, the gain between the

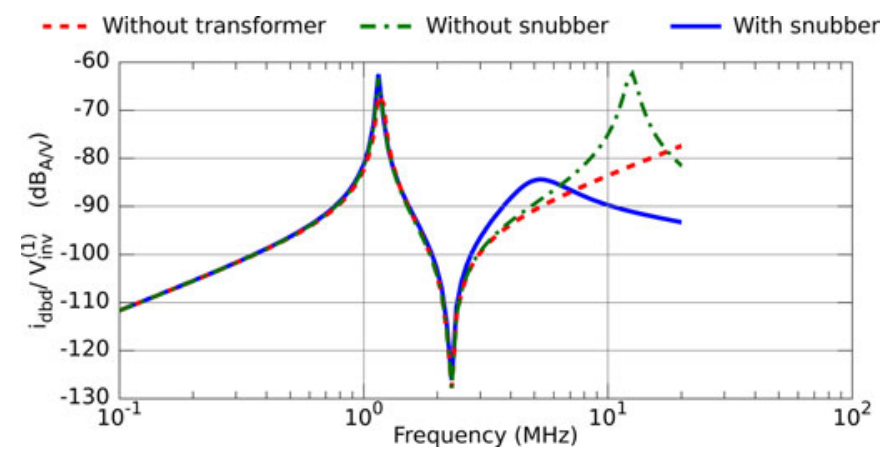

Fig. 14. Transformer influence on the frequency response.

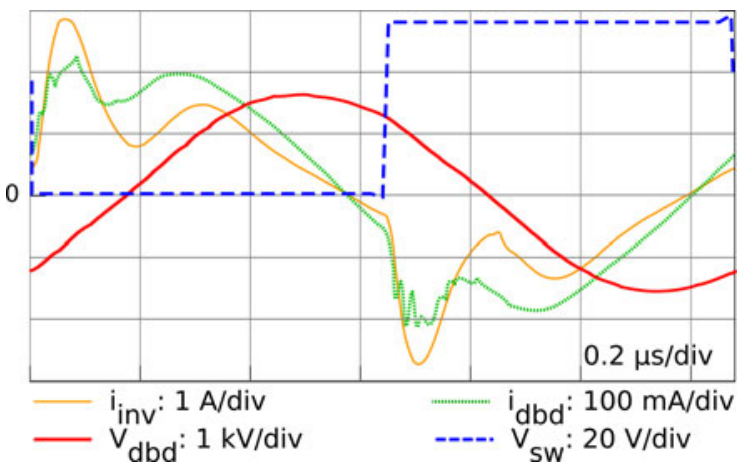

Fig. 15. Experimental waveforms for $V_{\mathrm{in}}=55 \mathrm{~V}$, using a high voltage probe.

DBD current $i_{\mathrm{dbd}}$ and the input voltage $\left(\hat{V}_{\mathrm{inv}}^{(1)}\right)$ is plotted. It is shown that the insertion of the medium voltage transformer brings out a high-frequency mode at $12 \mathrm{MHz}$ (see Fig. 14) superimposed to the first resonant mode $(1.2 \mathrm{MHz})$. In order to attenuate this HF mode, a snubber circuit $(22 \mathrm{pF}-1 \mathrm{k} \Omega)$ is added in parallel of the transformer secondary winding.

\section{B. Measurements}

1) Waveforms: The experimental waveforms where a high voltage probe is used are plotted in Fig. 15. The operating frequency is $781 \mathrm{kHz}$, and the injected power $P_{\mathrm{dbd}}$ is $39 \mathrm{~W}$. One can note that the voltage probe used to measure $V_{\mathrm{dbd}}$ influences the system behavior since its equivalent parasitic capacitance $(8 \mathrm{pF})$ is placed in parallel to the DBD device. When no highvoltage probe is inserted, the resonant frequency is $949 \mathrm{kHz}$ instead of $781 \mathrm{kHz}$ (see Fig. 16).

2) Switching Conditions: A low switching time is reached for the experiments: $3 \mathrm{~ns}$ for switching $\mathrm{ON}$ and $5 \mathrm{~ns}$ for switching OFF (see Fig. 16). Moreover, no voltage overshoot is measured. It proves the quality of the PCB design. In addition, the softswitching behavior is reached since the inverter current $i_{\text {inv }}$ is about a few hundreds of milliampere during the blocking time.

\section{CONCLUSION}

In the framework of DBD power supplies design, the interest of the high-frequency operating conditions has been shown: it allows us to supply DBD devices with short discharge current at 


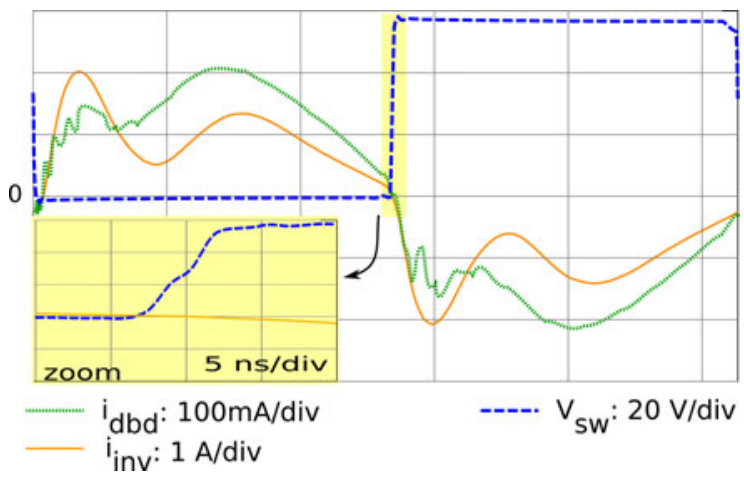

Fig. 16. Experimental waveforms for $V_{\text {in }}=55 \mathrm{~V}$ without high voltage probe and zoom on the blocking time.

a high repetition rate and to perform an accurate control of the injected power. This study has shown too, that theoretically, a transformerless resonant topology is appropriate in that regard. The use of wide bandgap switches and an appropriate PCB design allows us to reach a high operating frequency, which could be probably even higher than presented in this study. Moreover, thanks to this topology, working with a good $P_{\mathrm{dbd}} /\left(\hat{V}_{\mathrm{in}} \hat{i}_{\mathrm{in}}\right)$ ratio is possible, leading to compact power converters. It is also shown that the temperature drift has to be compensated.

Nevertheless, the frequency rise brings to light new challenges. The resonant inductor design becomes a more sensitive issue which limits the operating frequency. Indeed, highfrequency phenomena strongly increase the loss factor which results in an efficiency decrease and leads either to increase the input voltage and the switches voltage rating, or either to use a medium voltage transformer. The actual resonant inductor exhibits a loss factor which has to be reduced even more if higher frequencies have to be reached and the medium voltage transformer has to be eliminated. Moreover, parasitic elements such as the equivalent parallel parasitic capacitance of the inductor start playing an important role above the mega-Hertz range. That element should also be taken into account during the inductor designing process.

\section{ACKNOWLEDGMENT}

The authors would like to thank the French-Colombian cooperation (ECOS Nord, COLCIENCIAS-ICETEX program) and to the French Midi-Pyrénées region for support.

\section{REFERENCES}

[1] U. Kogelschatz. (2003). Dielectric-barrier discharges: Their history, discharge physics, and industrial applications. Plasma Chemistry Plasma Process. [Online]. 23, pp. 1-46, 10.1023/A:1022470901385. [Online]. Available: http://dx.doi.org/10.1023/A:1022470901385

[2] F. Massines, A. Rabehi, P. Decomps, R. B. Gadri, P. Segur, and C. Mayoux, "Experimental and theoretical study of a glow discharge at atmospheric pressure controlled by dielectric barrier," J. Appl. Phys., vol. 83, no. 6, pp. 2950-2957, Mar. 1998.

[3] F. Massines, G. Gouda, N. Gherardi, M. Duran, and E. Croquesel. (2001). The role of dielectric barrier discharge atmosphere and physics on polypropylene surface treatment. Plasmas Poly- mers [Online]. 6, pp. 35-49, 10.1023/A:1011365306501. Available: http://dx.doi.org/10.1023/A:1011365306501

[4] R. Seeböck, H. Esrom, M. Charbonnier, M. Romand, and U. Kogelschatz. (2001). Surface modification of polyimide using dielectric barrier discharge treatment. Surf. Coatings Technol. [Online]. 142144, pp. 455-459, Proc. 7th Int. Conf. Plasma Surf. Eng. Available: http://www.sciencedirect.com/science/article/pii/S0257897201010854

[5] C. Zhang, T. Shao, K. Long, Y. Yu, J. Wang, D. Zhang, P. Yan, and Y. Zhou, "Surface treatment of polyethylene terephthalate films using DBD excited by repetitive unipolar nanosecond pulses in air at atmospheric pressure," IEEE Trans. Plasma Sci., vol. 38, no. 6, pp. 1517-1526, Jun. 2010.

[6] G. Vezzu, J. Lopez, A. Freilich, and K. Becker, "Optimization of largescale ozone generators," IEEE Trans. Plasma Sci., vol. 37, no. 6, pp. 890-896, Jun. 2009.

[7] C. Ordiz, J. Alonso, M. Costa, J. Ribas, and A. Calleja, "Development of a high-voltage closed-loop power supply for ozone generation," in Proc. 23rd Апnи. IEEE Appl. Power Electron. Conf. Expo., Feb. 2008, pp. 1861-1867.

[8] M. Lomaev, E. Sosnin, V. Tarasenko, D. Shits, V. Skakun, M. Erofeev, and A. Lisenko. (2006). Capacitive and barrier discharge excilamps and their applications (review). Instrum. Exp. Tech. [Online]. 49, pp. 595-616, 10.1134/S0020441206050010. Available: http://dx.doi.org/10.1134/S0020441206050010

[9] L. Chang, T. Guo, J. Liu, C. Zhang, Y. Deng, and X. He, "Analysis and design of a current-source CLCC resonant converter for DBD applications," IEEE Trans. Power Electron., vol. 29, no. 4, pp. 1610-1621, Apr. 2014.

[10] D. Florez, R. Diez, and H. Piquet, "Dcm operated series resonant inverter for the supply of dbd excimer lamps," IEEE Trans. Ind. Appl., vol. PP, no. 99, p. 1, 2013.

[11] M. Amjad, Z. Salam, M. Facta, and S. Mekhilef, "Analysis and implementation of transformerless LCL resonant power supply for ozone generation," IEEE Trans. Power Electron., vol. 28, no. 2, pp. 650-660, Feb. 2013.

[12] D. Florez, R. Diez, K. Hay, and H. Piquet, "Dbd excimer lamp power supply with fully controlled operating conditions," in Proc. 13th Int. Conf. Optim. Electr. Electron. Equipment, 2012, pp. 1346-1352.

[13] X. Bonnin, H. Piquet, N. Naudé, M. Chérif Bouzidi, N. Gherardi, and J.-M. Blaquière, "Design of a current converter to maximize the power into homogeneous dielectric barrier discharge (DBD) devices," Eur. Phys. J. Appl. Phys., vol. 64, Oct. 2013.

[14] J. M. Williamson, D. D. Trump, P. Bletzinger, and B. N. Ganguly. (2006). Comparison of high-voltage AC and pulsed operation of a surface dielectric barrier discharge. J. Phys. D, Appl. Phys. [Online]. 39(20), p. 4400. [Online]. Available: http://stacks.iop.org/0022-3727/39/i=20/a=016

[15] L. T. Doanh, S. Bhosle, G. Zissis, and H. Piquet, "Estimation of the light output power and efficiency of a xecl dielectric barrier discharge exciplex lamp using one-dimensional drift-diffusion model for various voltage waveforms," IEEE Trans. Ind. Appl., vol. 49, no. 1, pp. 331-340, Jan./Feb. 2013.

[16] J. Rao, K. Liu, and J. Qiu, "A novel all solid-state sub-microsecond pulse generator for dielectric barrier discharges," IEEE Trans. Plasma Sci., vol. 41, no. 3, pp. 564-569, Mar. 2013.

[17] W. Shin, J. Choi, and T. Kim, "Bidirectional pulse plasma power supply for treatment of air pollution," in Proc. 37th IEEE Power Electron. Spec. Conf., 2006, pp. 1-6.

[18] R.-L. Lin, Y.-F. Chen, and Y.-Y. Chen, "Analysis and design of selfoscillating full-bridge electronic ballast for metal halide lamp at 2.65mhz operating frequency," IEEE Trans. Power Electron., vol. 27, no. 3, pp. 1589-1597, Mar. 2012.

[19] R. Díez, J. Salanne, H. Piquet, S. Bhosle, and G. Zissis, "Predictive model of a dbd lamp for power supply design and method for the automatic identification of its parameters," Eur. Phys. J. Appl. Phys., vol. 37, pp. 307$313,2007$.

[20] N. Naudé, J.-P. Cambronne, N. Gherardi, and F. Massines. (2005). Electrical model and analysis of the transition from an atmospheric pressure townsend discharge to a filamentary discharge. J. Phys. D, Appl. Phys. [Online]. 38(4), p. 530. Available: http://stacks.iop.org/0022$3727 / 38 / \mathrm{i}=4 / \mathrm{a}=004$

[21] J. Brandelero, B. Cougo, T. Meynard, and N. Videau, "A non-intrusive method for measuring switching losses of gan power transistors," in Proc. 39th Annu. Conf. IEEE Ind. Electron. Soc., Nov. 2013, pp. 244-249.

[22] M. Rodriİguez, A. Rodriİguez, P. Miaja, D. Lamar, and J. Zuniga, "An insight into the switching process of power mosfets: An improved analytical 
losses model," IEEE Trans. Power Electron., vol. 25, no. 6, pp. 1626-1640, Jun. 2010.

[23] D. Reusch, F. Lee, D. Gilham, and Y. Su, "Optimization of a high density gallium nitride based non-isolated point of load module," in Proc. IEEE Energy Convers. Congr. Expo., 2012, pp. 2914-2920.

[24] J. Wang, H.-H. Chung, and R.-H. Li, "Characterization and experimental assessment of the effects of parasitic elements on the mosfet switching performance," IEEE Trans. Power Electron., vol. 28, no. 1, pp. 573-590, Jan. 2013.

[25] D. Reusch and J. Strydom, "Understanding the effect of PCB layout on circuit performance in a high frequency gallium nitride based point of load converter," in Proc. 28th Annu. IEEE Appl. Power Electron. Conf. Expo., 2013, pp. 649-655.

[26] V. Lopez, A. Navarro-Crespin, R. Schnell, C. Branas, F. Azcondo, and R. Zane, "Current phase surveillance in resonant converters for electric discharge applications to assure operation in zero-voltage-switching mode," IEEE Trans. Power Electron., vol. 27, no. 6, pp. 2925-2935, Jun. 2012.
[27] N. Videau, J. Brandelero, T. Meynard, V. Bley, D. Flumian, G. Fontes, and E. Sarraute, "High switching frequency 5 -phase interleaved buck converter with gallium nitride transistors," in Proc. IEEE Workshop Wide Bandgap Power Devices Appl., 2013.

[28] EPC, EPC. [Online]. Available: http://epc-co.com/epc/Products/ eGaNFETs.aspx

[29] Aperam, datasheets. [Online]. Available: http://www.aperam.com/en/ alloys-amilly/downloads/datasheet/

[30] X. Bonnin, H. Piquet, R. Diez, and D. Florez, "Designing the high voltage transformer of power supplies for dbd: Windings arrangment to reduce the parasitic capacitive effects," in Proc. 15th Eur. Conf. Power Electron. Appl., 2013, pp. 1-9. 